\title{
Impact of Teaching on Surgical Site Infection after Colonic Surgery
}

\author{
Fabian Grass, MD, ${ }^{*}, 1$ Basile Pache, MD, ${ }^{*}, 1$ Christiane Petignat, MD, ${ }^{\dagger}$ Estelle Moulin, MD, ${ }^{\dagger}$ \\ Dieter Hahnloser, $M D,{ }^{*}$ Nicolas Demartines, $M D,{ }^{*}$ and Martin Hübner, $M D^{*}$
}

*Department of Visceral Surgery, Lausanne University Hospital CHUV, Lausanne, Switzerland; and
†Department of Hospital Preventive Medicine, Lausanne University Hospital CHUV, Lausanne, Switzerland

OBJECTIVE: The present study aimed to evaluate whether teaching had an influence on surgical site infections (SSI) after colonic surgery.

DESIGN: Colonic surgeries between January 2014 and December 2016 were retrospectively reviewed. Demographics, surgical details, and SSI rates were compared between teaching procedures vs. experts. Risk factors for SSI were identified by multinominal logistic regression.

SETTING: SSI were prospectively assessed by an independent National Surveillance Program (www.swissnoso.ch) at Lausanne University Hospital CHUV, a tertiary academic institution.

PARTICIPANTS: Included in the present analysis were patients documented in a prospective institutional enhanced recovery after surgery (ERAS) database and who were prospectively monitored by the independent National Infection Surveillance Committee between January 1, 2014 and December 31, 2016.

RESULTS: In all, 315 patients constituted the study cohort. Demographic and surgical items were comparable between teaching $(n=161)$ vs. expert operations $(n=135)$ except for higher occurrence of wound contamination class III-IV (13 vs. $19 \%, \mathrm{p}=0.046)$ in patients operated by experts. Overall, 61 patients (19\%) developed SSI, namely 25 patients $(16 \%)$ in the teaching group and 32 patients $(24 \%)$ in the expert group $(\mathrm{p}=0.077)$. Contamination class III-IV $(\mathrm{OR}=3.2 ; 95 \% \mathrm{CI}: 1.4-7.5, \mathrm{p}=0.005)$ and open surgery $(\mathrm{OR}=3.4 ; 95 \% \mathrm{CI}: 1.8-6.7, \mathrm{p}<0.001)$ were independent risk factors for SSI, while teaching had no significant impact $(\mathrm{OR}=0.6 ; 95 \% \mathrm{CI}$ : $0.3-1.2$, $\mathrm{p}=0.153)$.
CONCLUSIONS: Surgical teaching was feasible and safe after colonic surgery in the present cohort and had no impact on SSI rate. (J Surg Ed 75:1287-1291. () 2018 Association of Program Directors in Surgery. Published by Elsevier Inc. All rights reserved.)

KEY WORDS: colorectal surgery, teaching, surgical site infection, risk factors

COMPETENCIES: Practice-Based Learning and Improvement, Systems-Based Practice

\section{INTRODUCTION}

Teaching of young surgeons represents an important task of every academic hospital to ensure long-term quality of care. ${ }^{1,2}$ Skills of the operating surgeon are directly related to short-term outcome after surgery. ${ }^{3}$ Surgical site infections (SSI) represent the most frequent complication after colorectal surgery, even within enhanced recovery pathways. ${ }^{4}$

The aim of the present study was to evaluate the impact of teaching on SSI after colonic surgery in a tertiary academic institution.

\section{METHODS}

\section{Patients}

Included in the present analysis were patients documented in a prospective institutional database as outlined below and who were prospectively monitored by the independent National Infection Surveillance Committee (www.swissnoso.ch) between January 1, 2014 and December 31, 2016 at Lausanne University Hospital (CHUV). All patients were treated within a standardized ERAS pathway. Open and laparoscopic colectomies and colonic stoma procedures in an elective and emergency setting were retained. Excluded were patients who were not assessed by the national surveillance team. 
This study was considered a quality improvement project and approved by the Institutional Review Board (Commission cantonale d'éthique de la recherche sur l'être humain CER-VD \# 2016-00991). The study was conducted according to the STROBE criteria and registered under www.researchregistry.com (UIN researchregistry 2867).

Demographics and surgical information were prospectively assessed by the operating surgeon in a dedicated database; accuracy of data entry was cross-checked by the consultant surgeons (D.H and M.H.) and a dedicated ERAS nurse. Demographics included age, gender, Body Mass Index (BMI), American Society of Anesthesiologists (ASA) score, while comorbidities were assessed by the Charlson score. ${ }^{6}$ Further recorded were the presence of malignancy, immunosuppressive treatments (i.e., steroids) by the time of the procedure and previous abdominal surgery. Surgical information included type of procedure (colectomy: left, right (including segmental) or total (excluding proctocolectomy); stoma procedures: colostomy, colostomy closure and Hartmann reversal), approach (open vs. laparoscopy), conversion rate, setting (elective vs. emergency within 72 hours after unplanned admission), procedure duration (time from skin incision to skin closure), estimated blood loss based on surgeons and anesthesiologists' assessment, intraoperative transfusion and intraoperative complication (defined as adverse event during the procedure, which significantly extended procedure duration). According to ERAS protocol, mechanical oral bowel preparation was never performed. ${ }^{7}$ Patients undergoing leftsided colectomy were treated by rectal enemas the day before surgery and the morning of the day of surgery, while patients undergoing right-sided colectomy did not get any preparation.

According to institutional guidelines, intravenous cefuroxime $1.5 \mathrm{~g}$ and metronidazole $500 \mathrm{mg}$ were systematically administered 60-30 minutes before incision. As an alternative in case of non-tolerance, clindamycin $600 \mathrm{mg}$ and ciprofloxacin $400 \mathrm{mg}$ were used. Besides antibiotic prophylaxis, infection-preventing measures were adhered to according to NICE recommendations. ${ }^{8}$

\section{Teaching}

Senior surgeons (experts) were 3 board certified consultant surgeons (M.H., D.H., and ND) by the Society for Visceral Surgery (www.sgvc.ch). Teaching procedures were defined as procedures carried out to an extent of at least $75 \%$ by junior surgeons (fellows in colorectal surgery who had completed their general surgical training and performed at least 20 colonic resections and/or stoma procedures) under direct, continuous and close (face-to-face) supervision by one of the experts. All procedures were entirely standardized by the consultants. 9,10

\section{Assessment and classification of surgical site infection}

SSI were prospectively assessed, in-hospital and post-discharge [systematic phone call at postoperative day (POD) 30], by a national surveillance program by the independent committee (www.swissnoso.ch). Methodological details of this assessment have been published before. ${ }^{11}$ SSI were classified according to the Center for Disease Control (CDC) National Nosocomial Infection Surveillance (NNIS) criteria into superficial incisional, deep incisional, and organ space infections. ${ }^{12}$ Contamination class was assessed by the surgeon and classified at the end of the procedure as clean contaminated (grade II), contaminated (grade III), or infectious (grade IV). ${ }^{12}$

\section{Outcomes/study endpoints}

The primary binary endpoint was the comparison teaching vs. expert. In a second step, uni- and multivariate risk factors for SSI were identified.

\section{Statistical analysis}

Descriptive statistics for categorical variables were reported as frequency (\%), while continuous variables were reported as mean (standard deviation). Chi-square was used for comparison of categorical variables. All statistical tests were two-sided and a level of 0.05 was used to indicate statistical significance. Variables with p-values $\leq 0.1$ were then entered into a multivariate logistic regression (based on a probit regression model) to provide adjusted estimations of the odds ratio (OR). Data analysis was performed with the Statistical Software for the Social Sciences SPSS Advanced Statistics 22 (IBM Software Group, 200W. Madison St., Chicago, IL 60606, USA).

\section{RESULTS}

Three hundred and fifteen patients with complete datasets were retained for the present analysis. Nineteen patients (6\%) were excluded due to inability to clearly assign them to 1 of the 2 groups (teaching vs. expert) according to the definition, leaving 296 patients for final analysis. One hundred and sixty-one procedures $(55 \%)$ were performed by fellows (teaching procedures), while 135 surgeries (45\%) were carried out by colorectal consultants (experts). Demographic and surgical details are displayed in Table 1.

Sixty-one patients (19\%) developed SSI during the 30-day observation period. Of these, $16(26 \%)$ presented with superficial incisional, 7 (12\%) with deep incisional and 38 (62\%) with organ space infection. SSI occurred at POD $10 \pm 6$. No significant difference with regarding incidence of SSI was noted between the two comparative groups $(\mathrm{p}=0.077)$ (Table 2$)$. 
TABLE 1. Baseline and Surgical Characteristics

\begin{tabular}{|c|c|c|c|c|}
\hline & $\begin{array}{c}\text { All patients } \\
(n=315)\end{array}$ & $\begin{array}{l}\text { Teaching } \\
(n=161)\end{array}$ & $\begin{array}{c}\text { Expert } \\
(n=135)\end{array}$ & $\mathbf{p}$ \\
\hline $\begin{array}{l}\text { Age [years], (mean } \pm \mathrm{SD}) \\
\text { Gender, male }(\%) \\
\text { BMI }\left[\mathrm{kg} / \mathrm{m}^{2}\right],(\text { mean } \pm \mathrm{SD})\end{array}$ & $\begin{array}{c}65 \pm 17 \\
166(53) \\
25.6 \pm 6.2\end{array}$ & $\begin{array}{c}65 \pm 17 \\
82 \pm(51) \\
24.9 \pm 5.5\end{array}$ & $\begin{array}{c}64 \pm 16 \\
72 \pm(53) \\
26.4 \pm 6.9\end{array}$ & $\begin{array}{l}0.592 \\
0.727 \\
0.066\end{array}$ \\
\hline A HA & $173(55)$ & $90(56)$ & $74(55)$ & 0.898 \\
\hline III-IV & $142(45)$ & 71 (44) & $61(45)$ & \\
\hline Charlson $\geq 3(\%)$ & $60(19)$ & $30(19)$ & $27(20)$ & 0.769 \\
\hline Immunosuppression (\%) & 32 (10) & $16(10)$ & $14(10)$ & 1.000 \\
\hline Previous abdominal surgery (\%) & $170(54)$ & $91(57)$ & $68(51)$ & 0.346 \\
\hline & $161(51)$ & 79 (49) & 72 (53) & 0.803 \\
\hline \multicolumn{5}{|l|}{ Procedure $(\%)$} \\
\hline $\begin{array}{l}\text { Left colectomy } \\
\text { Right colectomy }\end{array}$ & $\begin{array}{l}132(42) \\
91(29)\end{array}$ & $\begin{array}{l}70(44) \\
44(28)\end{array}$ & $\begin{array}{l}55(41) \\
41(30)\end{array}$ & $\begin{array}{l}0.724 \\
0.699\end{array}$ \\
\hline Total colectomy & $13(4)$ & $5(3)$ & $7(5)$ & 0.237 \\
\hline Other colectomy & $25(8)$ & $12(7)$ & $10(8)$ & 0.805 \\
\hline Colostomy & $16(5)$ & $10(6)$ & $6(4)$ & 0.778 \\
\hline Colostomy closure & $16(5)$ & $10(6)$ & $6(4)$ & 0.778 \\
\hline Hartmann reversal & $22(7)$ & $10(6)$ & $10(8)$ & 0.636 \\
\hline Minimal invasive surgery (\%) & $201(64)$ & $102(63)$ & $86(64)$ & 1.000 \\
\hline Emergency surgery (\%) & $101(32)$ & $45(28)$ & $49(36)$ & 0.127 \\
\hline Operating time [minutes], (mean $\pm \mathrm{SD}$ ) & $170 \pm 80$ & $170 \pm 70$ & $170 \pm 90$ & 0.844 \\
\hline \multicolumn{5}{|l|}{ Contamination class (\%) } \\
\hline || & $261(83)$ & $140(87)$ & $105(78)$ & 0.046 \\
\hline III-IV & $54(17)$ & $21(13)$ & $30(22)$ & \\
\hline Conversion (\%) & $41(13)$ & $11(11)$ & $13(15)$ & 0.375 \\
\hline Intraoperative complication (\%) & $32(10)$ & $14(9)$ & 17 (13) & 0.327 \\
\hline Blood loss [mL], (mean \pm SD) & $100 \pm 190$ & $90 \pm 130$ & $130 \pm 240$ & 0.109 \\
\hline Intraoperative transfusion (\%) & $16(5)$ & $6(4)$ & $10(7)$ & 0.112 \\
\hline Interval $A B$ administration-incision $30-60 \mathrm{~min}(\%)$ & $148(47)$ & 81 (49) & 59 (44) & 0.403 \\
\hline
\end{tabular}

BMI-body mass index, ASA-American Society of Anaesthesiologists, Charlson-Charlson comorbidity score, Contamination class: II-clean contaminated, III-contaminated, IV-infectious, AB-antibiotic.

Significant values are indicated in bold characters.

\section{Factors associated with SSI}

Univariate factors associated with SSI were minimally invasive surgery $(37 \%$ in patients with SSI vs. $72 \%$ in patients without SSI, $\mathrm{p}=<0.001)$, emergency surgery ( $45 \%$ vs. $30 \%, \mathrm{p}=0.057)$, teaching $(44 \%$ vs. $57 \%, \mathrm{p}=$ 0.077 ), intraoperative complication (19\% vs. $8 \%, p=$ 0.025 ) and contamination class III-IV (39\% vs. $12 \%$, $\mathrm{p}=<0.001)$. Multivariate risk factors for SSI are displayed in the Figure contamination class III-IV $(\mathrm{OR}=3.2$; $95 \%$ CI: $1.4-7.5, p=0.005)$ constituted an independent risk factor. On the other hand, minimally invasive surgery (OR
$=0.3 ; 95 \%$ CI; $0.1-0.6, \mathrm{p}<0.001)$ protected against SSI. Teaching had no impact on development of SSI $(\mathrm{OR}=0.6$; 95\% CI: 0.3-1.2, $\mathrm{p}=0.153$ ).

\section{DISCUSSION}

In the present cohort of 296 patients undergoing a case mix of colonic procedures within enhanced recovery care, surgical teaching had no impact on SSI. Both groups (teaching vs. expert) were comparable regarding pertinent surgical parameters including operation time, conversion

TABLE 2. Incidence of Surgical Site Infection

\begin{tabular}{lcccc}
\hline & All patients $(\boldsymbol{n}=\mathbf{3 1 5})$ & Teaching $(\boldsymbol{n}=\mathbf{3 1 5})$ & Expert $(\boldsymbol{n}=\mathbf{3 1 5})$ & $\mathbf{p}$ \\
\hline Total number of SSI (\%) & $61(19)$ & $25(16)$ & $32(24)$ & 0.077 \\
Superficial incisional $(\%)$ & $16(5)$ & $4(3)$ & $11(8)$ & $\mathbf{0 . 0 3 3}$ \\
Deep incisional (\%) & $7(2)$ & $2(1)$ & $4(3)$ & 0.417 \\
Organ space infection $(\%)$ & $38(12)$ & $19(12)$ & $17(13)$ & 0.860 \\
\hline
\end{tabular}

SSI-surgical site infection.

Significant values are indicated in bold characters. 


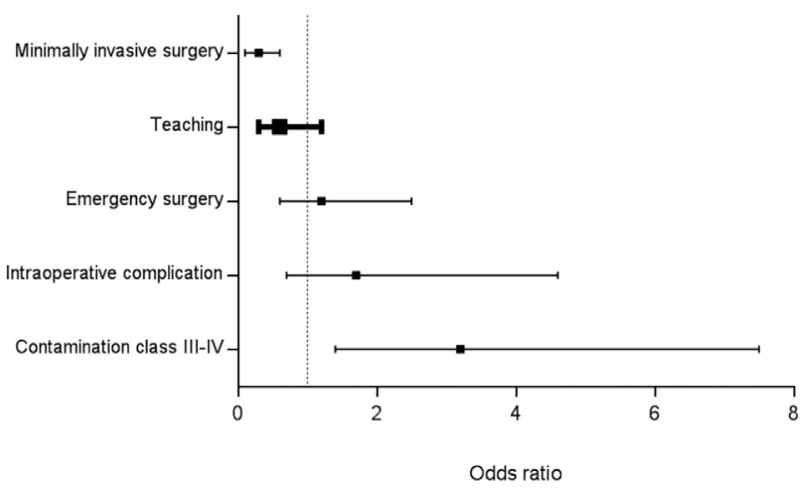

FIGURE. Multivariate analysis. Displayed as odds ratio with 95\% confidence interval. Contamination class: III-contaminated, IV-infectious.

rate, and intraoperative complications. Hence, teaching was feasible and safe in the present academic institution, without compromising the assessed main outcome SSI.

Every academic facility should aim to train young surgeons without compromising patients' outcome. Former studies have assessed the influence of supervised surgical training in open colorectal surgery on operating time and surgical outcome, suggesting training to be feasible providing appropriate teaching conditions. ${ }^{2,13}$ Laparoscopy has been implemented as standard technique for most colorectal resections due to benefits regarding pain, functional recovery and length of stay without compromising oncological outcome. ${ }^{14}$ However, laparoscopic procedures represent a technical challenge for young surgeons, pictured by a substantial learning curve. ${ }^{15}$ In a structured training setting, laparoscopic surgery including cancer surgery was safely performed by supervised trainees with equal short-term outcome compared to consultants' performance. ${ }^{16,17}$ Conversion rates and oncological adequacy of resection did not differ between fellows and staff surgeons in another quality improvement project. ${ }^{18}$ Finally, a recent meta-analysis of 19 observational studies showed equal results regarding oncological long-term outcomes. ${ }^{19}$

The results of the present study confirm these findings in a Swiss teaching facility with a heterogeneous case mix of colonic surgeries where all procedures were carried out in a standardized, structured teaching environment. Standardization of surgical procedures is a way to faster implementation of technical skills. ${ }^{20}$ Only 3 colorectal consultants with similar teaching strategies were in charge of training over the study period, probably contributing to equal outcome in both settings in the present cohort.

The present study focused on SSI, the most frequent complication after colorectal surgery. The observed rate of $19 \%$ compares to former reports, but undoubtedly represents a challenge for caregivers of the present institution. ${ }^{21,22}$ Meticulous prospective and systematic assessment of SSI by the independent national surveillance committee might partly explain the rather high SSI rate.
From the authors' perspective, bundled infection-preventing interventions, best possible compliance to enhanced recovery protocol with periodic audits and prospective quality improvement projects might be ways to decrease SSI in the future. ${ }^{23,24}$

Several limitations of the present study need to be mentioned, beyond inherent limitations of a retrospective study. Teaching was defined as at least $75 \%$ of the procedure performed by trainees. However, as a general rule, more challenging procedure steps or critical situations tend to be managed by experts, which might constitute an intrinsic selection bias. This assumption is emphasized by higher contamination class in the expert group. Some patients might have been missed by merging of the two independent databases. However, no deliberate selection was performed and all non-selected patients recorded by the 2 sources were included in the study. Potential type II error due to modest sample size impeded analysis of subgroups and thus further conclusions. The patient cohort was rather heterogeneous regarding surgical procedures. However, due to modest patient accrual in Switzerland, the results might be representative for daily clinical practice in an academic teaching hospital.

In conclusion, teaching had no influence on surgical site infection after colonic surgery. A structured, standardized environment allowed for equal results comparing trainees and experts.

\section{ACKNOWLEDGMENTS}

The authors would like to acknowledge the support provided by all members of the Enhanced Recovery After Surgery team in Lausanne, in particular Valérie Addor, dedicated ERAS nurse.

The authors declare to have no conflicts of interests and no sources of support and funding for this work.

\section{REFERENCES}

1. Renwick AA, Bokey EL, Chapuis PH, et al. Effect of supervised surgical training on outcomes after resection of colorectal cancer. Br J Surg. 2005;92(5):631-636.

2. Montroni I, Ghignone F, Rosati G, et al. The challenge of education in colorectal cancer surgery: a comparison of early oncological results, morbidity, and mortality between residents and attending surgeons performing an open right colectomy. J Surg Educ. 2014;71(2):254-261.

3. Sorensen LT, Malaki A, Wille-Jorgensen P, et al. Risk factors for mortality and postoperative complications after gastrointestinal surgery. J Gastrointest Surg. 2007;11(7):903-910. 
4. Forbes SS, Stephen WJ, Harper WL, et al. Implementation of evidence-based practices for surgical site infection prophylaxis: results of a pre- and postintervention study. J Am Coll Surg. 2008;207(3):336-341.

5. Roulin D, Donadini A, Gander S, et al. Costeffectiveness of the implementation of an enhanced recovery protocol for colorectal surgery. $\mathrm{Br} J$ Surg. 2013;100(8):1108-1114.

6. de Groot V, Beckerman H, Lankhorst GJ, Bouter LM. How to measure comorbidity: a critical review of available methods. J Clin Epidemiol. 2003;56(3): 221-229.

7. Gustafsson UO, Scott MJ, Schwenk W, et al. Guidelines for perioperative care in elective colonic surgery: Enhanced Recovery After Surgery (ERAS((R))) Society recommendations. World J Surg. 2013;37(2):259-284.

8. Leaper D, Burman-Roy S, Palanca A, et al. Prevention and treatment of surgical site infection: summary of NICE guidance. Br Med J. 2008;337:a1924.

9. Hubner M, Larson DW, Wolff BG. How I do itradical right colectomy with side-to-side stapled ileocolonic anastomosis. J Gastrointest Surg. 2012;16(8): 1605-1609.

10. Kummer A, Slieker J, Grass F, Hahnloser D, Demartines N, Hubner M. Enhanced recovery pathway for right and left colectomy: comparison of functional recovery. World J Surg. 2016;40(10):2519-2527.

1 1. Staszewicz W, Eisenring MC, Bettschart V, Harbarth S, Troillet N. Thirteen years of surgical site infection surveillance in Swiss hospitals. J Hosp Infect. 2014;88 (1):40-47.

12. Emori TG, Culver DH, Horan TC, et al. National nosocomial infections surveillance system (NNIS): description of surveillance methods. Am J Infect Control. 1991;19(1):19-35.

13. Borowski DW, Ratcliffe AA, Bharathan $B$, et al. Involvement of surgical trainees in surgery for colorectal cancer and their effect on outcome. Colorectal Dis. 2008;10(8):837-845.

14. Goh BK. Impact of laparoscopic resection for colorectal cancer on operative outcomes and survival. Ann Surg. 2007;246(2):338-339 author reply 9-40.
15. Tekkis PP, Senagore AJ, Delaney CP, Fazio VW. Evaluation of the learning curve in laparoscopic colorectal surgery: comparison of right-sided and left-sided resections. Ann Surg. 2005;242(1):83-91.

16. Krishna A, Russell M, Richardson GL, Rickard MJ, Keshava A. Supervised surgical training and its effect on the short-term outcome in laparoscopic colorectal surgery. Colorectal Dis. 2013;15(8):e483-e487.

17. Langhoff PK, Schultz M, Harvald T, Rosenberg J. Safe laparoscopic colorectal surgery performed by trainees. J Surg Educ. 2013;70(1):144-148.

18. Verheijen PM, vd Ven AW, Davids PH, Clark DA, Pronk A. Teaching colorectal surgery in the laparoscopic era; is it safe? J Surg Educ. 2010;67(4): 217-221.

19. Kelly M, Bhangu A, Singh P, Fitzgerald JE, Tekkis PP. Systematic review and meta-analysis of trainee- versus expert surgeon-performed colorectal resection. $\mathrm{Br} J$ Surg. 2014;101(7):750-759.

20. Page AJ, Gani F, Crowley KT, et al. Patient outcomes and provider perceptions following implementation of a standardized perioperative care pathway for open liver resection. Br J Surg. 2016;103(5):564-571.

21. Gomila A, Badia JM, Carratala J, et al. Current outcomes and predictors of treatment failure in patients with surgical site infection after elective colorectal surgery. A multicentre prospective cohort study. J Infect. 2017;74(6):555-563.

22. Gronnier C, Grass F, Petignat $C$, et al. Influence of enhanced recovery pathway on surgical site infection after colonic surgery. Gastroenterol Res Pract. 2017;2017:9015854.

23. Cima R, Dankbar E, Lovely J, et al. Colorectal surgery surgical site infection reduction program: a national surgical quality improvement program-driven multidisciplinary single-institution experience. J Am Coll Surg. 2013;216(1):23-33.

24. Jurt J, Slieker J, Frauche P, et al. Enhanced recovery after surgery: can we rely on the key factors or do we need the bel ensemble? World J Surg. 2017;41 (10):2464-2470. 